\title{
WEAK ROTUNDITY IN BANACH SPACES
}

\author{
A. C. YORKE \\ (Received 8 December 1976) \\ Communicated by E. Strzelecki
}

\begin{abstract}
Weak rotundity is defined. One version characterizes the duals of very smooth spaces, another characterizes the duals of extremely smooth spaces. Weak rotundity methods are used to investigate the differentiability of the norm in $E^{* *}$, and to obtain information about quotient spaces, when $E^{* *}$ is smooth.
\end{abstract}

It is well known that in general smoothness and rotundity are dual concepts only in reflexive spaces. However, Cudia (1964) has developed a type of rotundity which is dual to smoothness, even in the non-reflexive case. Recently, Sullivan (1975) has introduced two new types of smoothness, extreme smoothness and very smoothness. The purpose of the first part of this paper is to construct rotundity conditions which characterize the duals of these spaces.

In Section 1 the rotundity of Cudia is redefined in terms of sequences, and renamed weak rotundity. It is shown that one type of weak rotundity characterizes the duals of very smooth spaces, while another characterizes the duals of extremely smooth spaces. In Section 2, weak rotundity methods are used to investigate the F-differentiable and UG-differentiable analogs of very smoothness and extreme smoothness. Section 3 obtains some information about quotient spaces.

This paper is based on part of a doctoral thesis submitted to the University of Newcastle under the supervision of Associate Professor J. R. Giles.

\section{Weak rotundity}

Notation. $E$ denotes a real Banach space, $E^{*}$ its dual; $\hat{E}$ is the natural embedding of $E$ in $E^{* *} ; S(E), S\left(E^{*}\right)$ and $S(\hat{E})$ denote the unit spheres of $E, E^{*}$ and $\hat{E}$, respectively; $D_{E}: E \rightarrow 2^{E^{*}}$ is the duality map which assigns to each $x \in E$ the set $\left\{f \in E^{*}:\|f\|=\|x\|\right.$ and $\left.f(x)=\|f\|\|x\|\right\} ; Y^{\perp}$ denotes $\left\{f \in E^{*}: f(y)=0\right.$ for all $\left.y \in Y\right\}$.

As in Sullivan (1975), for $x, y \in S(E)$, let $\rho(x, y)=\|x+y\|+\|x-y\|-2$. The norm of $E$ is Gâteaux differentiable (G-differentiable) at $x \in S(E)$ in the $y \in S(E)$ 
direction if for each $\lambda$,

$$
\lim _{\lambda \rightarrow 0} \rho\left(\frac{x, \lambda y}{\lambda}\right)=0 .
$$

In this case, $E$ will be said to be $G$-differentiable at $x$ in the $y$ direction. If $E$ is G-differentiable at $x$ in the $y$ direction for every $y \in S(E), E$ is $G$-differentiable at $x$. If $E$ is $\mathrm{G}$-differentiable for every $x \in S(E), E$ is $G$-differentiable. $E$ is Fréchet differentiable ( $F$-differentiable) at $x \in S(E)$ if

$$
\lim _{\lambda \rightarrow 0} \sup _{\|y\|=1}\left\{\rho\left(\frac{x, \lambda y}{\lambda}\right)\right\}=0 .
$$

$E$ is $F$-differentiable if $E$ is F-differentiable at every $x \in S(E)$. $E$ is uniformly Gâteaux differentiable (UG-differentiable) in the $y \in S(E)$ direction if

$$
\lim _{\lambda \rightarrow 0} \sup _{\|x\|=1}\left\{\rho\left(\frac{x, \lambda y}{\lambda}\right)\right\}=0 \text {. }
$$

If $E$ is UG-differentiable in the $y$ direction for every $y \in S(E), E$ is $U G$-differentiable.

$E$ is smooth if $D_{E}(x)$ is a singleton for each $x \in S(E) . E$ is G-differentiable at $x \in S(E)$ if and only if $E$ is smooth at $x$ (see Giles, 1971, p. 109). $E$ is rotund if $x, y \in S(E)$ with $\|x+y\|=2$, implies that $x=y$; that is, $E$ is rotund if $S(E)$ contains no line segments. If $E^{*}$ is smooth (rotund), then $E$ is rotund (smooth), but in general this cannot be reversed.

$E$ is very smooth if $D_{E^{* *}}(\hat{x})$ is a singleton for each $x \in S(E)$. $E$ is extremely smooth if $F \in S\left(E^{* *}\right)$ and $\mathfrak{F},\left(\mathfrak{H} \in S\left(E^{* * *}\right)\right.$ with $\mathfrak{F}(F)=1=\mathfrak{G}(F)$, implies that $\mathfrak{F}-\mathfrak{G} \in E^{\perp}$. $E$ is extremely smooth if and only if $E^{* *}$ is G-differentiable in the $S(\hat{E})$ directions 'see Sullivan, 1975, p. 16).

' Let $f, g \in S\left(E^{*}\right)$. $E$ is said to be weakly rotund at $f$ in the $g$ direction if for any tequences $\left\{x_{n}\right\},\left\{y_{n}\right\}$ in $S(E)$ such that $f\left(\frac{1}{2}\left(x_{n}+y_{n}\right)\right) \rightarrow 1$, then $g\left(x_{n}-y_{n}\right) \rightarrow 0$ as $\rightarrow \rightarrow \infty$. If $A$ and $B$ are non-empty subsets of $S\left(E^{*}\right)$, then $E$ is weakly rotund at $A$ $M$ the $B$ directions if $E$ is weakly rotund at $f$ in the $g$ direction for each $f \in A$ and $\in B$. In this paper, the sets $A$ and $B$ will both be $S\left(E^{*}\right)$ when $E$ is being considered, While for $E^{*}$, the sets $A$ and $B$ may be either $S(\hat{E})$ or $S\left(E^{* *}\right)$. If $E$ is weakly rotund pniformly at $S\left(E^{*}\right)$ in the $S\left(E^{*}\right)$ directions, $E$ is weakly uniformly rotund (WUR). If $E$ is weakly rotund at $S\left(E^{*}\right)$ uniformly in the $S\left(E^{*}\right)$ directions, $E$ is uniformly wtund $(U R)$ at $S\left(E^{*}\right)$. When $E^{*}$ is weakly rotund uniformly at $S\left(E^{* *}\right)$ in the $S(\hat{E})$ rections, $E^{*}$ is said to be weak-* uniformly rotund $\left(W^{*} U R\right)$.

Therefore, including uniformity in both "slots", there are four types of weak fotundity for $E$ and sixteen for $E^{*}$. However, the following proposition shows that at most nine of these are distinct.

Proposition 1. Let $F \in S\left(E^{* *}\right)$. Then

(i) $E^{*}$ is weakly rotund at $F$ uniformly in the $S\left(E^{* *}\right)$ directions if and only if $E^{*}$ is weakly rotund at $F$ uniformly in the $S(\hat{E})$ directions; 
(ii) $E^{*}$ is weakly rotund uniformly at $S\left(E^{* *}\right)$ in the $F$ direction if and only if $E^{*}$ is weakly rotund uniformly at $S(\hat{E})$ in the F-direction.

Proof. (i) Let $\left\{f_{n}\right\},\left\{g_{n}\right\}$ be sequences in $S\left(E^{*}\right)$ with $F\left(\frac{1}{2}\left(f_{n}+g_{n}\right)\right) \rightarrow 1$ as $n \rightarrow \infty$. Then $\left\|f_{n}-g_{n}\right\| \rightarrow 0$ if and only if $\hat{x}\left(f_{n}-g_{n}\right) \rightarrow 0$ uniformly for all $\hat{x} \in S(\hat{E})$. But this is equivalent to $G\left(f_{n}-g_{n}\right) \rightarrow 0$ uniformly for all $G \in S\left(E^{* *}\right)$, hence the result.

(ii) is proved similarly.

Proposition 2.

(i) $E$ is smooth if and only if $E^{*}$ is weakly rotund at $S(\hat{E})$ in the $S(\hat{E})$ directions.

(ii) $E$ is very smooth if and only if $E^{*}$ is weakly rotund at $S(\hat{E})$ in the $S\left(E^{* *}\right)$ directions.

(iii) $E$ is extremely smooth if and only if $E^{*}$ is weakly rotund at $S\left(E^{* *}\right)$ in the $S(\hat{E})$ directions.

Proo . All three proofs are similar so only the proof of (ii) will be given. Assume that $E$ not very smooth at some $\hat{x} \in S(\hat{E})$. Then the norm of $\hat{E}$ is not G-differentiable at $\hat{x}$ in the $F$-direction, for some $F \in S\left(E^{* *}\right)$. Hence there is an $\varepsilon>0$, and a sequence of positive real numbers $\left\{\lambda_{n}\right\}$, with $\lambda_{n} \rightarrow 0$, such that $0<\varepsilon \leqslant \rho\left(\left(\hat{x}, \lambda_{n} F\right) / \lambda_{n}\right)$. Since $\lambda_{n} \rightarrow 0$ as $n \rightarrow \infty$, both $\left\|\hat{x}+\lambda_{n} F\right\|$ and $\left\|\hat{x}-\lambda_{n} F\right\| \rightarrow 1$. Thus there are sequences $\left\{f_{n}\right\}$ and $\left\{g_{n}\right\}$ in $S\left(E^{*}\right)$ such that

$$
\left(\hat{x}+\lambda_{n} F\right)\left(f_{n}\right)>\left\|\hat{x}+\lambda_{n} F\right\|-\lambda^{2}
$$

and

$$
\left(\hat{x}-\lambda_{n} F\right)\left(g_{n}\right)>\left\|\hat{x}+\lambda_{n} F\right\|-\lambda_{n}^{2}
$$

for all $n$. Therefore

$$
\begin{aligned}
0<\varepsilon & \leqslant \rho\left(\frac{\hat{x}, \lambda_{n} F}{\lambda_{n}}\right)=\frac{\left\|\hat{x}+\lambda_{n} F\right\|+\left\|\hat{x}-\lambda_{n} F\right\|-2}{\lambda_{n}} \\
& <\frac{\left(\hat{x}+\lambda_{n} F\right)\left(f_{n}\right)+\left(\hat{x}-\lambda_{n} F\right)\left(g_{n}\right)-2+2 \lambda_{n}^{2}}{\lambda_{n}} \\
& =\frac{\hat{x}\left(f_{n}+g_{n}\right)+\lambda_{n} F\left(f_{n}-g_{n}\right)-2+2 \lambda_{n}^{2}}{\lambda_{n}} \leqslant F\left(f_{n}-g_{n}\right)+2 \lambda_{n} .
\end{aligned}
$$

Now $\hat{x}\left(f_{n}+g_{n}\right) \rightarrow 2$, but $F\left(f_{n}-g_{n}\right)>\varepsilon-2 \lambda_{n}$ for all $n$. Therefore $F\left(f_{n}-g_{n}\right)$ remains bounded away from zero, hence $E^{*}$ is not weakly rotund at $\hat{x}$ in the $F$-direction.

Conversely if $E^{*}$ is not weakly rotund at $\hat{x}$ in the $F \in S\left(E^{* *}\right)$ direction, there is an $\varepsilon>0$, and sequences $\left\{f_{n}\right\},\left\{g_{n}\right\}$ in $S\left(E^{*}\right)$, such that $\hat{x}\left(f_{n}+g_{n}\right) \rightarrow 2$ but $F\left(f_{n}-g_{n}\right) \geqslant \varepsilon$ for all $n$. Let $\mathfrak{F}$ and $\left(\mathfrak{G}\right.$ be $\sigma\left(E^{* * *}, E^{* *}\right)$ cluster points of $\left\{\hat{f}_{n}\right\}$ and $\left\{\hat{g}_{n}\right\}$, respectively. 
Then $\|\mathfrak{F}\| \leqslant 1$ and $\|\mathfrak{5}\| \leqslant 1$. But $\hat{x}\left(f_{n}\right)$ and $\hat{x}\left(g_{n}\right)$ both converge to 1 as $n \rightarrow \infty$, so $\mathfrak{F}(\hat{x})=\mathfrak{G}(\hat{x})=1$. Thus $\|\mathfrak{F}\|=\|\mathfrak{F}\|=1$. But since $(\mathfrak{F}-(\mathfrak{G})(F) \geqslant \varepsilon>0$, $\mathfrak{F}$ cannot be equal to $(\mathfrak{5}$. Thus $E$ is not very smooth at $x$.

Comparing Proposition 2(i) with Cudia (1964, p. 294) gives that the weak rotundity described here is equivalent to Cudia's rotundity.

Clearly, both very smoothness and extreme smoothness imply smoothness, with all three of these concepts being equivalent in reflexive spaces. However, if $E$ is not reflexive, then very smoothness and extreme smoothness are not comparable, as (ii) and (iii) of Proposition 2 show.

Proposition 3. The following are equivalent:

(i) $E$ is weakly rotund at $S\left(E^{*}\right)$ in the $S\left(E^{*}\right)$ directions;

(ii) $E^{*}$ is smooth;

(iii) $E^{* *}$ is weakly rotund at $S\left(\hat{E}^{*}\right)$ with $S\left(\hat{E}^{*}\right)$ directions.

Proof. (i) implies (ii). If $E^{*}$ is not smooth there is an $\varepsilon>0$, a sequence of positive real numbers $\left\{\lambda_{n}\right\}$, with $\lambda_{n} \rightarrow 0$, and $f, g \in S\left(E^{*}\right)$ such that

$$
0<\varepsilon \leqslant \rho\left(\frac{f, \lambda_{n} g}{\lambda_{n}}\right)=\frac{\left\|f+\lambda_{n} g\right\|+\left\|f-\lambda_{n} g\right\|-2}{\lambda_{n}} .
$$

Now proceed as in Proposition 2. Let $\left\{x_{n}\right\}$ and $\left\{y_{n}\right\}$ in $S(E)$ be such that $\left(f+\lambda_{n} g\right)\left(x_{n}\right)>\left\|f+\lambda_{n} g\right\|-\lambda_{n}^{2}$ and $\left(f-\lambda_{n} g\right)\left(y_{n}\right)>\left\|f-\lambda_{n} g\right\|-\lambda_{n}^{2}$ for each $n$. Then

$$
0<\varepsilon \leqslant \rho\left(\frac{f, \lambda_{n} g}{\lambda_{n}}\right)<\frac{f\left(x_{n}+y_{n}\right)+\lambda_{n} g\left(x_{n}-y_{n}\right)-2+2 \lambda_{n}^{2}}{\lambda_{n}} \leqslant g\left(x_{n}-y_{n}\right)+2 \lambda_{n} .
$$

Hence $g\left(x_{n}-\lambda_{n}\right)$ remains bounded away from zero, even though $f\left(x_{n}+y_{n}\right) \rightarrow 2$ as $n \rightarrow \infty$. Thus (i) fails to hold.

(ii) implies (iii) by Proposition 2(i).

(iii) implies (i) obviously.

Therefore the weak rotundity of Proposition 2(i) and smoothness are dual concepts, even in non-reflexive spaces (see Cudia, 1964, p. 294).

Proposition 2 shows that one version of weak rotundity gives a characterization of the dual spaces of very smooth spaces, while another version characterizes the duals of extremely smooth spaces. Proposition 3(i) shows that no amount of weak rotundity on $E$, without uniformity in one of the "slots", is strong enough to imply very smoothness or extreme smoothness in $E^{*}$ if $E$ is not reflexive. Therefore the weak rotundity of Proposition 2(ii) and very smoothness are dual concepts only in the reflexive case. This remark also holds for the weak rotundity of Proposition 2(iii) and extreme smoothness. 
Proposition 4. If $E$ is UG-differentiable, then $E$ is extremely smooth.

Proof. $E$ is UG-differentiable if and only if $E^{*}$ is W*UR (see Giles, 1976, p. 395). If $E^{*}$ is $\mathrm{W}^{*} \mathrm{UR}$, then $E^{*}$ is weakly rotund at $S\left(E^{* *}\right)$ in the $S(\hat{E})$ directions. The result now follows by applying Proposition 2(iii).

Zizler (1969, p. 203) has given an example of a non-reflexive smooth space which is not UG-differentiable. In Giles (1976, p. 407) there is an example of a reflexive smooth space, hence extremely smooth space, which is not UG-differentiable.

\section{Differentiability of the norm in $\mathbf{E}^{* *}$}

It follows from the definition that $E^{*}$ being very smooth is equivalent to $\hat{E}^{*}$ being smooth in $E^{* * *}$. Since this implies that $E$ is reflexive, if $E$ is non-reflexive there must be a point of non-smoothness on $S\left(\hat{E}^{*}\right)$ (Giles, 1974, p. 118). However, since every F-differentiable space is very smooth (Giles, 1975), a space may be smooth at every $\hat{x} \in S(\hat{E})$ and not reflexive. In fact, Smith (1976) has given an example of a non-reflexive space which is smooth at every $F \in S\left(E^{* *}\right)$.

This section looks at the F-differentiability and UG-differentiability of the embedding $\hat{E}$ in $E^{* *}$, by considering the F-differentiable and UG-differentiable analogs of very smoothness and extreme smoothness.

Proposition 5. $E$ is F-differentiable at $x \in S(E)$ if and only if $E^{*}$ is $U R$ at $\hat{x}$.

Proof. Assume that $E$ is not F-differentiable at $x$, and let $\left\{f_{n}\right\},\left\{g_{n}\right\},\left\{\lambda_{n}\right\}$, and let $\varepsilon$ be as in Proposition 2. Then

$$
\begin{aligned}
0<\varepsilon & <\sup _{\|y\|=1}\left\{\frac{\left\|x+\lambda_{n} y\right\|+\left\|x-\lambda_{n} y\right\|-2}{\lambda_{n}}\right\} \\
& \leqslant \sup _{\|\hat{y}\|=1}\left\{\frac{\hat{x}\left(f_{n}+g_{n}\right)+\lambda_{n} \hat{y}\left(f_{n}-g_{n}\right)-2+2 \lambda_{n}^{2}}{\lambda_{n}}\right\} \\
& \leqslant \sup _{\|\hat{\nu}\|=1}\left\{\hat{y}\left(f_{n}-g_{n}\right)+2 \lambda_{n}\right\} .
\end{aligned}
$$

Therefore although $\hat{x}\left(f_{n}+g_{n}\right) \rightarrow 2$, as $n \rightarrow \infty,\left\|f_{n}-g_{n}\right\|$ remains bounded away from zero for all $n$. Thus $E^{*}$ is not UR at $\hat{x}$.

This argument is reversible. If $E^{*}$ is not UR at $\hat{x}$ there are sequences $\left\{f_{n}\right)$ and $\left\{g_{n}\right\}$ in $S\left(E^{*}\right)$ such that $\hat{x}\left(f_{n}+g_{n}\right) \rightarrow 2$, but $\left\|f_{n}-g_{n}\right\| \geqslant \varepsilon$ for some $\varepsilon>0$. Since $\hat{x}\left(f_{n}+g_{n}\right) \rightarrow 2$, there is a sequence of positive real numbers $\left\{\lambda_{n}\right\}$, with $\lambda_{n} \rightarrow 0$, with 
the property that $2-\hat{x}\left(f_{n}+g_{n}\right) \leqslant \lambda_{n}^{2}$ for all $n$. Thus

$$
\begin{aligned}
\varepsilon-\lambda_{n} & \leqslant \frac{\hat{x}\left(f_{n}+g_{n}\right)-2+\lambda_{n}\left\|f_{n}-g_{n}\right\|}{\lambda_{n}} \\
& \leqslant \sup _{\|y\|=1}\left\{\frac{\hat{x}\left(f_{n}+g_{n}\right)+\lambda_{n} \hat{y}\left(f_{n}-g_{n}\right)-2}{\lambda_{n}}\right\} \\
& \leqslant \sup _{\|y\|=1} \rho\left(\frac{x, \lambda_{n} y}{\lambda_{n}}\right) .
\end{aligned}
$$

Hence

$$
\lim _{\lambda_{n} \rightarrow 0} \sup _{\|y\|=1} \rho\left(\frac{x, \lambda_{n} y}{\lambda_{n}}\right) \neq 0
$$

so $E$ is not F-differentiable at $x$.

This shows that $E$ is F-differentiable if and only if $E^{*}$ is UR at $S(\hat{E})$. Therefore this type of weak rotundity and F-differentiability are dual concepts, as Proposition 5 and the next proposition show (see Cudia, 1964, p. 296).

Proposition 6. Let $f \in S\left(E^{*}\right)$. Then the following are equivalent:

(i) $E$ is UR at f;

(ii) $E^{*}$ is F-differentiable at $f$;

(iii) $E^{* *}$ is UR at $f$.

Using the methods of Proposition 5, the proof is similar to Proposition 3, so will be omitted.

Now consider the F-differentiable analog of very smoothness; that is, the F-differentiability of $\hat{E}$ in $E^{* *}$.

Proposition 7. $E$ is $F$-differentiable at $x \in S(E)$ if and only if $\hat{E}$ is $F$-differentiable at $x$.

Proof. By Proposition 5 it is sufficient to prove that if $E^{*}$ is UR at $\hat{x}$, then $\hat{E}$ is F-differentiable at $\hat{x}$. If $\hat{E}$ is not F-differentiable at $\hat{x} \in S(\hat{E})$, there is an $\varepsilon>0$ and a sequence of positive real numbers $\left\{\lambda_{n}\right\}$, with $\lambda_{n} \rightarrow 0$, such that

$$
\limsup _{\lambda_{n} \rightarrow 0}\left\{\rho\left(\frac{\hat{x}, \lambda_{n} F}{\lambda_{n}}\right): F \in S\left(E^{* *}\right)\right\} \geqslant \varepsilon>0 .
$$

Now proceed as in Propositions 2 and 5. This gives

$$
\sup \left\{\frac{\hat{x}\left(f_{n}+g_{n}\right)+\lambda_{n} F\left(f_{n}-g_{n}\right)-2+2 \lambda_{n}^{2}}{\lambda_{n}}: F \in S\left(E^{* *}\right)\right\} \geqslant \varepsilon>0 .
$$

Once again $\hat{x}\left(f_{n}+g_{n}\right) \rightarrow 2$ as $n \rightarrow \infty$, but $\left\|f_{n}-g_{n}\right\|$ remains bounded away from zero. Hence $E^{*}$ is not UR at $\hat{x}$.

Therefore $\hat{E}$ is F-differentiable exactly when $E$ is F-differentiable. 
If $E$ is very smooth, then $\hat{E}$ may only be G-differentiable in $E^{*}$. Therefore Proposition 7 shows that the embedding of an F-differentiable space inherits a stronger property than the embedding of a very smooth space.

Next look at the F-differentiable analog of extreme smoothness, the F-differentiability of $E^{* *}$ in the $S(\hat{E})$ directions.

Proposition 8. $E^{* *}$ is $F$-differentiable at $G \in S\left(E^{* *}\right)$ if and only if $E^{* *}$ is $F$-differentiable at $G$ in the $S(\hat{E})$ directions.

Proof. By Proposition 6, $E^{* *}$ is F-differentiable at $G$ if and only if $E^{*}$ is UR at $G$. Therefore it is sufficient to show that if $E^{* *}$ is F-differentiable at $G$ in the $S(\hat{E})$ directions, then $E^{*}$ is UR at $G$. If $E^{*}$ is not UR at $G$ there is an $\varepsilon>0$, and sequences $\left\{f_{n}\right\}$ and $\left\{g_{n}\right\}$ in $S\left(E^{*}\right)$, such that $G\left(f_{n}+g_{n}\right) \rightarrow 2$, but $\left\|f_{n}-g_{n}\right\| \geqslant \varepsilon$ for all $n$. Let $\left\{\lambda_{n}\right\}$ be a sequence of positive real numbers, with $\lambda_{n} \rightarrow 0$, such that $2-G\left(f_{n}+g_{n}\right) \leqslant \lambda_{n}^{2}$ for all $n$. Then

$$
\begin{aligned}
\varepsilon-\lambda_{n} & \leqslant \sup _{\|\hat{x}\|=1}\left\{\frac{G\left(f_{n}+g_{n}\right)+\lambda_{n} \hat{x}\left(f_{n}-g_{n}\right)-2}{\lambda_{n}}\right\} \\
& \leqslant \sup _{\|\hat{x}\|=1} \rho\left(\frac{G, \lambda_{n} \hat{x}}{\lambda_{n}}\right) .
\end{aligned}
$$

Therefore

$$
\lim _{\lambda_{n} \rightarrow 0} \sup _{\|\hat{x}\|=1} \rho\left(\frac{G, \lambda_{n} \hat{x}}{\lambda_{n}}\right) \neq 0,
$$

so $E^{* *}$ cannot be F-differentiable at $G$ in the $S(\hat{E})$ directions.

Thus $E^{* *}$ need only be F-differentiable in the $S(\hat{E})$ directions to imply that $E$ is reflexive.

So $E$ is "very F-differentiable" exactly when $E$ is F-differentiable, while $E$ is "extremely F-differentiable" is equivalent to $E^{* *}$ being F-differentiable. Therefore, in one sense, "very F-differentiability" is too "weak" to be a distinct concept, while "extreme F-differentiability" is too "strong".

Now consider the UG-differentiable case. It is implicit in Theorem 2 of Giles (1976) that $E^{* *}$ is UG-differentiable if and only if the embedding $\hat{E}$ is UGdifferentiable in $E^{* *}$. Thus $E$ is "very UG-differentiable" if and only if $E^{* *}$ is UG-differentiable. The next result, therefore, is not surprising.

Proposition 9. Let $y \in S(E) . E^{* *}$ is UG-differentiable in the $\hat{y}$ direction if and only if $E$ is $U G$-differentiable in the $y$ direction.

Proof. Since the latter condition is equivalent to $E^{*}$ being WUR in the $\hat{y}$ direction (Giles, 1976, p. 395), it is sufficient to show that if $E^{*}$ is WUR in the 
$\hat{y}$ direction, $E^{* *}$ is UG-differentiable in the $\hat{y}$ direction. If $E^{* *}$ is not UG-differentiable in the $\hat{y} \in S(\hat{E})$ direction, there is an $\varepsilon>0$ and a sequence of positive real numbers $\left\{\lambda_{n}\right\}$, with $\lambda_{n} \rightarrow 0$, such that

$$
0<\varepsilon \leqslant \lim _{\lambda_{n} \rightarrow 0} \sup \left\{\rho\left(\frac{F, \lambda_{n} \hat{y}}{\lambda_{n}}\right): F \in S\left(E^{* *}\right)\right\} .
$$

Now letting $\left\{f_{n}\right\}$ and $\left\{g_{n}\right\}$ be as in Proposition 2,

$$
0<\varepsilon \leqslant \sup \left\{\frac{F\left(f_{n}+g_{n}\right)+\lambda_{n} \hat{y}\left(f_{n}-g_{n}\right)-2+2 \lambda_{n}^{2}}{\lambda_{n}}: F \in S\left(E^{* *}\right)\right\} \text {. }
$$

Now let $n \rightarrow \infty$. Then $\hat{y}\left(f_{n}-g_{n}\right)$ remains bounded away from zero, even though $\left\|f_{n}+g_{n}\right\| \rightarrow 2$. Hence $E^{*}$ is not WUR in the $\hat{y}$ direction.

Thus $E$ is "extremely UG-differentiable" if and only if $E$ is UG-differentiable. So in contrast to the F-differentiable case, "very UG-differentiability" is too "strong" to be a distinct concept, while "extremely UG-differentiable" is too "weak".

Zizler $(1968$, p. 424$)$ has shown that any separable dual space admits an equivalent UG-differentiable norm. Thus $c_{0}^{*}=l_{1}$ admits an equivalent norm such that $l_{\infty}^{*}$ is UG-differentiable in the $S\left(\hat{l}_{1}\right)$ directions. However, $l_{\infty}^{*}=c_{0}^{* * *}$ must be nonsmooth for some $\hat{f} \in S\left(\hat{l}_{1}\right)$, since $c_{0}$ is not reflexive.

\section{Quotient spaces}

In this section, the term "subspace" means "norm closed subspace". For a subspace $L$ of $E$, the quotient space $E / L$ is the space of cosets of $L$ in $E$, with the norm $\|x+L\|=\inf \{\|x+l\|: l \in L\}$. If every two-dimensional quotient space of $E$ is smooth (rotund), then $E$ is smooth (rotund), but the converse is not true, unless $E^{*}$ is rotund (smooth) (Day, 1973, p. 145). Day (1973) has given an example of a rotund space with a non-rotund quotient space. Klee $(1959$, p. 62) has shown that any separable space admits a smooth norm such that $E / L$ is non-smooth, where $L$ is any non-reflexive subspace of $E$ with $\operatorname{dim} E / L \geqslant 2$.

This section uses weak rotundity to look at the quotient spaces of extremely smooth and very smooth spaces. First, however, it is interesting to see how much weak rotundity is needed on $E^{*}$ to imply rotundity.

Proposition 10. If $E^{*}$ is weakly rotund at $S\left(E^{* *}\right)$ in the $S(\hat{E})$ direction, then $E^{*}$ is rotund.

Proof. Assume that $E^{*}$ is not rotund. Then there are $f, g \in S\left(E^{*}\right)$ with $f \neq g$ such that $\|f+g\|=2$. By the Hahn-Banach Theorem there is an $F \in S\left(E^{* *}\right)$ with $F(f+g)=2$. But since $f \neq g$, there is an $\hat{x} \in S(E)$ with $\hat{x}(f-g) \geqslant \varepsilon>0$ for some $\varepsilon>0$. Thus $E^{*}$ is not weakly rotund at $F$ in the $\hat{x}$ direction. 
Note that Klee's (1959) example shows that in $E^{*}$ weak rotundity at $S(\hat{E})$ in the $S(\hat{E})$ direction is strictly weaker than rotundity.

Proposition 11. Let $Y$ be a subspace of $E^{*}$. Then if $E^{*}$ is weakly rotund at $S\left(E^{* *}\right)$ in the $S\left(E^{* *}\right)$ directions, $Y$ is weakly rotund at $S\left(Y^{*}\right)$ in the $S\left(Y^{*}\right)$ directions.

Proof. If $Y$ is not weakly rotund at $S\left(Y^{*}\right)$ in the $S\left(Y^{*}\right)$ directions, there is an $\varepsilon>0, F, G \in S\left(Y^{*}\right)$ and sequences $\left\{f_{n}\right\},\left\{g_{n}\right\}$ in $S(Y)$ such that $F\left(f_{n}+g_{n}\right) \rightarrow 2$, but $G\left(f_{n}-g_{n}\right) \geqslant \varepsilon$ for all $n$. By an application of the Hahn-Banach Theorem, there are functionals $F_{1}, G_{1} \in S\left(E^{* *}\right)$ such that $F_{1}=F$ and $G_{1}=G$ on $Y$. Hence $F_{1}\left(f_{n}+g_{n}\right) \rightarrow 2$, but $G_{1}\left(f_{n}-g_{n}\right) \geqslant \varepsilon$ for all $n$. Thus $E^{*}$ is not weakly rotund at $F_{1}$ in the $G_{1}$ direction.

If $E^{* *}$ is smooth, then $E^{*}$ is rotund, so every quotient space of $E$ is smooth. The next proposition improves this.

Proposition 12. If $E^{* *}$ is smooth then every quotient space of $E$ is both very smooth and extremely smooth.

Proof. If $E^{* *}$ is smooth, then Proposition 3 gives that $E^{*}$ is weakly rotund at $S\left(E^{* *}\right)$ in the $S\left(E^{* *}\right)$ directions. Thus by Proposition 2, $E$ is both very smooth and extremely smooth. Let $L$ be any subspace of $E$. Then $(E / L)^{*}$ is isometrically isomorphic to $L^{\perp}$ (Day, 1973, p. 30). Since $L^{\perp}$ is a subspace of $E^{*}$, Proposition 10 gives that $L^{\perp}$ is weakly rotund at $S\left(\left(L^{\perp}\right)^{*}\right)$ in the $S\left(\left(L^{\perp}\right)^{*}\right)$ directions. Now applying Proposition 2 gives the result.

In conclusion, note that if $E$ is extremely smooth Proposition 9 implies that $E^{*}$ is rotund, so every quotient space of $E$ is smooth. However, this does not necessarily imply that every quotient space is extremely smooth.

\section{References}

D. F. Cudia (1964), "The geometry of Banach spaces. Smoothness", Trans. Amer. Math. Soc. 110, 284-314.

M. M. Day (1973), Normed Linear Spaces, 3rd ed. (Springer-Verlag).

J. R. Giles (1971), "On a characterization of differentiability of the norm of a normed linear space", J. Austral. Math. Soc. 12, 106-114.

J. R. Giles (1974), “A non-reflexive Banach space has non-smooth third conjugate space", Canad. Math. Bull. 17, 117-119.

J. R. Giles (1975), “On smoothness of the Banach space embedding”, Bull. Austral. Math. Soc. 13, 69-74.

J. R. Giles (1976), "Uniformly weak differentiability of the norm and a condition of Vlasov", J. Austral. Math. Soc. 21(A), 393-409.

V. Klee (1959), "Some new results on smoothness and rotundity in normed linear spaces", Math. Annalen, 139, 51-63.

M. A. Smith (1976), "A smooth non-reflexive second conjugate space", Bull. Austral. Math. Soc. 15, 29-31. 
F. Sullivan (1975), "Geometrical properties determined by the higher duals of a Banach space" (preprint).

V. Zizler (1968), "Banach spaces with differentiable norms", Comm. Math. Univ. Carol. 8, 3, 415-440.

V. Zizler (1969), "Some notes on various rotundity and smoothness properties of separable Banach spaces", Comm. Math. Univ. Carol. 10, 2, 195-206.

Department of Mathematics

University of Newcastle

Newcastle, NSW 2308

Australia 